\title{
EL DESARROLLO Y DOMINIO DE LAS COMPETENCIAS CIENTÍFICO - PROFESIONALES DEL DOCENTE UNIVERSITARIO
}

\author{
Elmina Rivadeneira Rodríguez ${ }^{1}$, Ricardo Silva Bustillos ${ }^{2}$ \\ Universidad Técnica de Machala (Ecuador) ${ }^{1}$ \\ Rector del Instituto Superior de Tecnología 17 de julio \\ Ciudad del Conocimiento -YACHAY (Ecuador) ${ }^{2}$ \\ elminar@hotmail.com ${ }^{1}$
}

Cómo citar este artículo: Rivadeneira, E. y Silva, R. (2015) El desarrollo y dominio de las competencias científico-profesionales del docente universitario. CUMBRES, Revista Científica. 1(1) 30 - 34

\section{RESUMEN}

La sociedad del conocimiento requiere de un profesional intelectualmente autónomo para decidir por sí mismo, con responsabilidad, empatía, valiéndose del propio conocimiento gestionado y con competencias para someterla críticamente sus aportaciones científicas y académicas de manera argumentada, trasmitiendo modos de actuación y procedimientos de aprendizaje para toda la vida. El docente propicia las relaciones e interacciones entre discente y el docente como organizador, orientador, facilitador; entre aprendientes y de todos estos con la sociedad a través del proceso de aprender a conocer (cognitivo), hacer (cognoscitivo) y ser (meta cognitivo). El cambio de actitud permitirá desarrollar y dominar las competencias: epistemológicasmetodológicas, comunicativas, tecnológicas, socio-profesionales, didáctico- pedagógicas en los docentes para conseguir: Una actitud creativa y respetuosa; fomentar un clima de empatía y de ecuanimidad frente a las diferentes opiniones; defender sus tesis con argumentos y aceptar el cuestionamiento; comprender que existen diferentes enfoques para acercarse al estudio del fenómeno

Palabras clave: Competencias científico, profesionales, docente, discente

\section{ABSTRACT}

The knowledge society requires an intellectually independent professional to decide for himself, with responsibility, empathy, using the managed self-knowledge and skills to critically submit their scientific and academic contributions in a reasoned manner, transmitting modes of intervention and Learning lifetime. The teacher fosters relationships and interactions between learner and teacher as organizer, counselor, facilitator; between all these learners and to society through the process of learning to know (cognitive) to (cognitive) and be (metacognitive). The change in attitude will develop and master skills: methodological, communicative, technological epistemological-, socio-professional, didactic teaching in teachers to get: A creative and respectful attitude; foster a climate of empathy and equanimity towards different opinions; defend their thesis with arguments and accept the challenge; understand that there are different approaches to approach the study of the phenomenon

Keywords: Scientific skills, professional, teacher, learner

\section{INTRODUCCIÓN}

La ciencia necesita de conocimientos innovadores que permita al ser humano solucionar problemas del presente siglo, por ello requiere de un profesional competente que 
pueda actuar con independencia, responsabilidad, empatía y autonomía para someter a criticas sus aportaciones científica y académica de manera argumentada en su actuación y procedimientos de aprendizaje para la vida (Ausubel,1983).

El desarrollo de este artículo, este se divide en tres partes. En la primera parte se tratan los aspectos que propician las relaciones e interacciones con los docentes y los discentes, a través de los procesos cognitivos, cognoscitivos y metacognitivos (Delors, 1996). En la segunda parte el docente investigador que es capaz de desarrollar las competencias: epistemológicas-metodológicas, comunicativas, tecnológicas, socio profesionales, didáctico-pedagógicas y, en la tercera parte se enuncia el perfil del docente investigador: reflexivo, crítico, creativo, respetuoso, organizado, persistente, motivador y sobre todo desarrollar el autocontrol y el autoconcepto para defender sus tesis con argumentos y aceptar el cuestionamiento; comprender que existen diferentes enfoques para acercarse al estudio del fenómeno; desarrollar un lenguaje científico cuando se pretende que también los hagan los discentes.

\section{Ámbitos de la competencia}

Para (Perrenoud, 2004) afirma que los seres humanos usan procesos mentales, un enfoque del mundo y competencias relacionadas, que conservan similitudes independientes del marco o actividad de los que se trate; operan como actores en campos sociales cuyo funcionamiento comparte algunas características entre sí. Dentro del mo-

Figura 1. Proceso transdiciplinario de la competencia

\begin{tabular}{|l|l|l|l|}
\hline Filosofía & $\begin{array}{l}\text { Saber } \\
\text { pensar }\end{array}$ & Curiosidad & $\begin{array}{l}\text { Búsqueda de la verdad, mediante la } \\
\text { indagación (Pensamiento) }\end{array}$ \\
\hline Psicología & Saber actuar & Motivación & $\begin{array}{l}\text { Tiene capacidad de resolver problemas, } \\
\text { para aprender a actuar (Estructuras } \\
\text { mentales) }\end{array}$ \\
\hline Lingüística & $\begin{array}{l}\text { Saber } \\
\text { comunicarse }\end{array}$ & Expresión & $\begin{array}{l}\text { Receptar y trasmitir información } \\
\text { (Comunicación) }\end{array}$ \\
\hline Sociología & $\begin{array}{l}\text { Saber } \\
\text { interactuar }\end{array}$ & Colectividad & $\begin{array}{l}\text { Construir relaciones sociales } \\
\text { equitativas que transformen espacios } \\
\text { (Sociedad) }\end{array}$ \\
\hline Economía & $\begin{array}{l}\text { Saber } \\
\text { producir }\end{array}$ & Distribución & $\begin{array}{l}\text { Producir, distribuir y satisfacer las } \\
\text { necesidades del ser humano (Producción) }\end{array}$ \\
\hline Laboral & Saber hacer & Teoría y práctica & $\begin{array}{l}\text { Combinación de capacidades, } \\
\text { habilidades y actitudes (Desempeños) }\end{array}$ \\
\hline
\end{tabular}

delo didáctico-pedagógico, se puede describir el proceso de las competencias, tal y como se muestra en la figura 1. (Proceso transdiciplinario de la competencia)

El modelo didáctico - pedagógico para la formación de las competencias investigativas está compuesto por tres dimensiones, con sus correspondientes indicadores como son: la curricular, la didáctica - pedagógico y la epistemología - metodológica, que actuando como sistema a lo largo del proceso formativo en las carreras, favorecerán el objetivo planificado.

\section{Pilares fundamentales de la educación}

Desde una visión muy universal, pueden tenerse en consideración los "cuatro pilares de educación" reconocidos por la UNESCO.

Aprender a conocer: Concertar entre una cultura general suficientemente amplia y los conocimientos particulares de las diferentes disciplinas, en torno a problemas e interrogantes concretos. Esto requiere aprender a aprender, con el fin de aprovechar las posibilidades que ofrece la educación a lo largo de la vida (Ausubel, 1983).

Aprender a hacer: Adquirir no sólo una certificación profesional, sino más bien competencias que capaciten al individuo para hacer frente a gran número de situaciones previstas e imprevistas y a trabajar en equipo en busca del conocimiento multidisciplinario, interdisciplinario y transdiciplinario (Morín, 2000).

Aprender a vivir juntos: Realizar proyectos comunes y prepararse para asumir y resolver los conflictos, respetando los valores del pluralismo, el entendimiento mutuo y la paz, a través de la comprensión del otro y de las formas de interdependencia. Desarrollo de las relaciones intrapersonales e interpersonales (Goleman, 1999).

Aprender a ser: Actuar con creciente capacidad de autonomía, de juicio y responsabilidad personal, para que florezca en mejor forma la propia personalidad. Con tal fin, no subestima ninguna posibilidad de cada individuo en su proceso educativo: competencias intelectuales (memorizar, razonar, comprender, etc.), comunicativas, afectivas, estéticas, físicas, entre otras (Delors, 1966).

El proceso de formación basado en competencias profesionales brinda un espacio para la construcción de manera integrada de conocimientos, habilidades y valores que se constituyen en cualidades del sujeto. Por otra parte es un proceso con un carácter eminentemente social que se desarrolla en un contexto interdisciplinar, multidisciplinar y transdisciplinar, que parte de una concepción participativa y es estructurado a partir de un contenido socialmente construido e históricamente desarrollado, todo lo que tiene como consecuencia la formación de nuevas cualidades 
en el hombre, que se traducen en competencias profesionales.

\section{Las competencias científicos- profesionales del do- cente universitario.}

La gestión de calidad basada en las competencias científicos - profesionales necesita asumir las diferencias entre el pensamiento complejo del pensamiento simplificador, en donde la única certidumbre es la incertidumbre (Tobón, 2004).

Figura 2. Competencias epistemológicas - metodológicas

\begin{tabular}{|l|l|l|l|}
\hline \multicolumn{5}{|c|}{ COMPETENCIAS } \\
\hline Competencias & \multicolumn{1}{|c|}{$\begin{array}{l}\text { Aprender } \\
\text { Cognitivo }\end{array}$} & \multicolumn{1}{|c|}{$\begin{array}{c}\text { Saber } \\
\text { Cognoscitivo }\end{array}$} & \multicolumn{1}{c|}{$\begin{array}{c}\text { Hacer } \\
\text { Metacognitivo }\end{array}$} \\
\hline $\begin{array}{l}\text { Gestionar } \\
\text { conocimientos }\end{array}$ & $\begin{array}{l}\text { Desarrollos teóricos } \\
\text { desde la teoría del } \\
\text { conocimiento de las } \\
\text { ciencias }\end{array}$ & $\begin{array}{l}\text { Aplicará en los } \\
\text { procesos de la } \\
\text { investigación }\end{array}$ & $\begin{array}{l}\text { Interactúa } \\
\text { interdisciplinariamente }\end{array}$ \\
\hline Experimentar & $\begin{array}{l}\text { Metodología analítica } \\
\text { y experimental de } \\
\text { investigación }\end{array}$ & $\begin{array}{l}\text { Gestionará } \\
\text { docencia la } \\
\text { investigación } \\
\text { aplicar en la } \\
\text { transformación } \\
\text { sostenible }\end{array}$ & $\begin{array}{l}\text { Actúa con autonomía } \\
\text { intelectual y sensibilidad } \\
\text { social }\end{array}$ \\
\hline $\begin{array}{l}\text { Solucionar } \\
\text { problemas }\end{array}$ & $\begin{array}{l}\text { Principios de la lógica, } \\
\text { la crítica científica }\end{array}$ & $\begin{array}{l}\text { Analizará } \\
\text { críticamente } \\
\text { metódicamente } \\
\text { contradicciones y } \\
\text { propone } \\
\text { soluciones }\end{array}$ & $\begin{array}{l}\text { Asume posición crítica } \\
\text { y constructiva ante } \\
\text { problemas de su campo } \\
\text { de actuación }\end{array}$ \\
\hline
\end{tabular}

Las competencias son un conjunto de habilidades, destrezas, actitudes, valores, conocimientos y estrategias que unidos nos ayudan a encontrar la solución, en forma flexible y autónoma, a los problemas que enfrentamos en nuestra vida cotidiana. Las competencias implican no sólo conocimientos específicos, sino el desarrollo y dominio de la capacidad de utilizarlos como herramientas para enfrentar situaciones problemáticas de la vida.Las competencias constituyen "Un sistema de conocimientos, habilidades, valores y cualidades de la personalidad que se movilizan en función de las necesidades individuales y sociales, así como de los motivos, intereses y actitudes del profesional,
Figura 3. Competencias comunicativas - tecnológicas

\begin{tabular}{|c|c|c|c|}
\hline \multicolumn{4}{|c|}{$\begin{array}{c}\text { COMPETENCIAS } \\
\text { Comunicativas - Tecnológicas }\end{array}$} \\
\hline Competencias & \begin{tabular}{l|l} 
Aprender \\
Cognitivo
\end{tabular} & $\begin{array}{c}\text { Saber } \\
\text { Cognoscitivo }\end{array}$ & $\begin{array}{c}\text { Hacer } \\
\text { Metacognitivo }\end{array}$ \\
\hline $\begin{array}{l}\text { Lengua } \\
\text { materna }\end{array}$ & 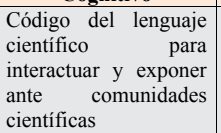 & $\begin{array}{lr}\text { Utilización } & \text { de } \\
\text { códigos de } & \text { la } \\
\text { apropiación } & y \\
\text { socialización } & \text { del } \\
\text { conocimiento } & \end{array}$ & $\begin{array}{l}\text { Actitud positiva y } \\
\text { solvencia comunicativa } \\
\text { en la interacción con } \\
\text { comunidades }\end{array}$ \\
\hline $\begin{array}{l}\text { Lengua } \\
\text { extranjera }\end{array}$ & $\begin{array}{l}\text { Lenguaje científico de } \\
\text { una segunda lengua de } \\
\text { amplia utilización en } \\
\text { su campo }\end{array}$ & $\begin{array}{lr}\text { Lee, interpreta } \text { y } \\
\text { escribe } & \text { textos } \\
\text { científicos } & \mathrm{e} \\
\text { interactúa } & \text { con } \\
\text { pares } & \\
\end{array}$ & $\begin{array}{l}\text { Actitud positiva y } \\
\text { solvencia comunicativa }\end{array}$ \\
\hline Argumentar & $\begin{array}{l}\text { Principios, métodos y } \\
\text { procedimientos de la } \\
\text { argumentación } \\
\text { científica }\end{array}$ & $\begin{array}{l}\text { Argumenta } \\
\text { científicamente } \\
\text { posición sobre sus } \\
\text { aportes }\end{array}$ & 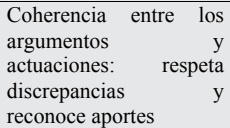 \\
\hline Tecnológicas & \begin{tabular}{lr}
\multicolumn{3}{l}{ Fundamentos, avances } \\
y alcances de \\
herramientas \\
tecnologías de punta
\end{tabular} & $\begin{array}{l}\text { Las aplica en el } \\
\text { proceso }\end{array}$ & $\begin{array}{l}\text { Posición abierta para su } \\
\text { incorporación en la } \\
\text { docencia e investigación }\end{array}$ \\
\hline
\end{tabular}

Figura 4. Competencias socio-profesionales y didáctico-pedagógicos

\begin{tabular}{|c|c|c|c|}
\hline \multicolumn{4}{|c|}{$\begin{array}{c}\text { COMPETENCIAS } \\
\text { Socio - Profesionales y Didácticas - Pedagógicas } \\
\end{array}$} \\
\hline Competencias & $\begin{array}{l}\text { Aprender } \\
\text { Cognitivo }\end{array}$ & $\begin{array}{c}\text { Saber } \\
\text { Cognoscitivo }\end{array}$ & $\begin{array}{c}\text { Hacer } \\
\text { Metacognitivo }\end{array}$ \\
\hline Liderazgo & $\begin{array}{l}\text { Teoría y dinámica del } \\
\text { trabajo colaborativo y } \\
\text { cooperativo }\end{array}$ & $\begin{array}{l}\text { Aplica estrategias } \\
\text { para el trabajo en } \\
\text { grupo, lidera } \\
\text { líneas, grupos y } \\
\text { proyectos }\end{array}$ & $\begin{array}{l}\text { Respeta diferencias en } \\
\text { el trabajo en grupo }\end{array}$ \\
\hline Autoformación & $\begin{array}{l}\text { Teorías, principios y } \\
\text { técnicas del } \\
\text { aprendizaje } \\
\text { permanente }\end{array}$ & $\begin{array}{l}\text { Accede a fuentes } \\
\text { de información y } \\
\text { las incorpora a su } \\
\text { desarrollo } \\
\text { científico }\end{array}$ & $\begin{array}{l}\text { Asimila el desarrollo de } \\
\text { la ciencia y } \\
\text { tecnología }\end{array}$ \\
\hline $\begin{array}{l}\text { Didáctica } \\
\text { pedagógicas }\end{array}$ & $\begin{array}{l}\text { Métodos } \mathrm{y} \\
\text { procedimientos del } \\
\text { aprender a aprender }\end{array}$ & $\begin{array}{l}\text { Dirigir el proceso } \\
\text { de enseñanza - } \\
\text { aprendizaje }\end{array}$ & \begin{tabular}{|l|} 
Promueve \\
aprendizaje \\
colaborativo, \\
cooperativo, \\
significativo \\
desarrollador \\
\end{tabular} \\
\hline
\end{tabular}


que permiten el desempeño satisfactorio en el ejercicio de la profesión; y que sólo pueden ser evaluadas a través del desempeño, considerando las exigencias sociales (Santos, 2005). El docente investigador debe desarrollar las competencias: figura 2 (epistemológicas-metodológicas), figura 3 (comunicativas-tecnológicas), figura 4 (socio profesionales y didáctico-pedagógicas).

El dominio cognitivo se relaciona con la capacidad de utilizar información para el pensamiento y el aprendizaje. El dominio metacognitivo tiene que ver con la habilidad de planificar, supervisar y evaluar.

\section{Cómo debe ser el docente universitario}

La ciencia requiere de nuevos conocimientos, es decir el docente y discente no solamente debe resolver problemas, también tiene que generar problemas científicos para poder aportar a la "sociedad de la producción del conocimiento y la creatividad" (Rivadeneira, 2015).

Para (Elton, 1987) afirma que el buen docente universitario a través de esta lista de adjetivos calificativos: bien organizado, bien preparado, interesado en la materia, amigable, flexible, servicial, creativo, claro, entusiasta, interesado en los estudiantes, abierto, sistemático, comprometido, entregado. (Ericksen, 1985) destaca los comportamientos y cualidades siguientes: selecciona y organiza el material del curso, guía a los estudiantes para el registro e integración de la información de modo que puedan recordarlo fácilmente, es competente en los procedimientos y métodos de su disciplina, mantiene la curiosidad intelectual de sus alumnos, promueve el aprendizaje independiente. (Ramsder, 1992) afirma que los profesores universitarios tienen estas características: Poseen un amplio repertorio de habilidades docente específicas, olvidan que su meta es el aprendizaje de los estudiantes, escuchan y aprenden de sus alumnos, evalúan constantemente su actuación docente, enseñan con entusiasmo, muestran preocupación y respeto por los alumnos, tienen facilidad para hacerse entender por los alumnos, hacen del estudiante un aprendiz autónomo, usan métodos que exigen al estudiante aprender activa y cooperativamente.

Para que un profesional se considere competente, no basta con lograr un desempeño eficiente, sino que es necesario que actué con compromiso, además dentro la competencia profesional integre en su estructura y funcionamiento elementos de orden cognitivo y motivacional que se expresen como una unidad reguladora en la actuación profesional (González, 2002).

\section{CONCLUSIONES}

Las nuevas tendencias en educación superior demandan un nuevo tipo de docente. La sociedad del conocimiento requiere de un profesional intelectualmente autónomo para decidir por sí mismo, con independencia, responsabilidad, empatía, valiéndose del propio conocimiento gestionado y con competencias para someter a crítica sus aportaciones científicas y académicas de manera argumentada, trasmitiendo modos de actuación y procedimientos de aprendizaje para toda la vida (Ausubel,1983)

El docente debe propiciar las relaciones e interacciones con los discentes, como: organizador, guía, orientador, facilitador, etc. Debe vincular todas estas interacciones con la sociedad a través del proceso de aprender a conocer (cognitivo), hacer (cognoscitivo) y ser (metacognitivo), para producir y reproducir la cultura en su sentido más amplio y lograr, sobre esa base, el cumplimiento de los objetivos desarrolladores y significativos. En síntesis, el nuevo docente requiere los siguientes atributos:

1. Dominio y desarrollo de las competencias cognitivas, cognoscitivas y metacognitivos, en concordancia con la aplicación de los cuatro pilares fundamentales saber conocer, saber hacer, saber convivir y saber ser.

2. Desarrollar y dominar las competencias: epistemológicas-metodológicas, comunicativas, tecnológicas, socio-profesionales, didáctico-pedagógicas.

3. Ser: reflexivo, critico, creativo, respetuoso, organizado, persistente, motivador y sobre todo desarrollar el autocontrol y el autoconcepto para defender sus tesis con argumentos y aceptar el cuestionamiento; comprender que existen diferentes enfoques para acercarse al estudio del fenómeno; desarrollar un lenguaje científico cuando se pretende que también los hagan los discentes.

No podemos construir la sociedad del Siglo XXI con las competencias docentes del Siglo XX.

\section{REFERENCIAS BIBLIOGRÁFICAS}

- Ausubel, D. (1983). Psicología educativa: Un punto de vista cognoscitivo. México: Editorial Trillas.

- Delors, J. (1966). Los cuatros pilares de la educación. En la educación encierra un tesoro. España: Informe a la UNESCO de la Comisión Internacional sobre la educación para el siglo XXI.

- Elton, L. (1987). Teaching in higher education: appraisal and training. London: Kogan Page. 
- Ericksen, S. (1985). The essence of good teaching: helping student to learn and remember what they learn. San Francisco: Jossey - bass.

- Goleman, D. (1999). La inteligencia emocional. Buenos aires: Javier Vergara.

- González, V. (2002). ¿Qué significa ser un profesional competente?: Reflexiones desde una perspectiva psicoIógica. Cuba: En Revista Cubana de Educación Superior.

- Morín, E. (2000). Los siete saberes necesarios para una educación del futuro. UNESCO.

- Perrenoud, PH. (2004). La clave de los campos sociales: competencias del autor autónomo. FCE, México.

- Ramsder, P. (1992). Learning to teach in higner education. Londres: London Routledge.

- Rivadeneira, E. (2014). Teoría de las competencias y competencias científicas de los docentes en Educación Superior. En Estados Unidos: Informe del Curso de Postdoctorado en competencias científico- profesionales.

- Rivadeneira, E. y Silva, R. (2015). Desarrollo y Dominio de las Competencias Científicos - Profesionales en la Educación Universitaria. En Ecuador: Ponencia en el I. Congreso de Ciencia y Tecnología UTMach 2015.

- Santos, J. (2005). Modelo pedagógico para el mejoramiento del desempeño pedagógico profesional de los profesores de agronomía de los Institutos Politécnicos Agropecuarios. En La Habana, Cuba: Tesis en opción al título de Dra. En Ciencias Pedagógicas. Instituto Superior Pedagógico Enrique José Varona.

- Tobón, S. (2004). Formación basada en competencias: pensamiento complejo, diseño curricular y didáctica. Bogotá. 